\title{
Innovation of E-Commerce Terminal Express Cooperative Distribution based on Big Data Platform
}

\author{
Zhipeng $\mathrm{Chu}^{\mathrm{a}}$ and Ping $\mathrm{Yu}^{\mathrm{b}}{ }^{\text {** }}$ \\ ${ }^{a}$ Ningbo Institute of Finance and Economics, Ningbo, 315175, China \\ ${ }^{b}$ School of Electrical Engineering and Information Technology, Changchun Institute of Technology, Changchun, 130012, China
}

\begin{abstract}
In order to strengthen the logistics distribution ability at the end of e-commerce, it is necessary to study the co-delivery collaborative delivery method of e-commerce. When the current delivery method is used to deliver the courier at the end of the e-commerce, the distribution takes a long time to meet the distribution requirements of the user, and there is a problem of low distribution efficiency and low customer satisfaction. On the basis of the big data platform, an e-commerce end express co-delivery distribution method is proposed. By calculating the inventory cost, fixed investment cost, storage cost and operation cost, the total cost of the distribution center is coordinated, and the lowest total cost is selected as the distribution center. The distribution time range is analyzed by the concept of time window to obtain a penalty function. According to the penalty function, the path optimization model of e-commerce end express delivery is established. According to the path optimization model, the optimal route of collaborative delivery is obtained, and the coordinated delivery of the e-commerce end express is completed. The experimental results show that the proposed method has high distribution efficiency and high customer satisfaction.
\end{abstract}

Keywords: big data platform; e-commerce; collaborative distribution; path optimization

(Submitted on November 6, 2018; Revised on December 5, 2018; Accepted on January 2, 2019)

(C) 2019 Totem Publisher, Inc. All rights reserved.

\section{Introduction}

E-commerce is also known as "online retailing", which means that businesses conduct electronic transactions with customers. The core strength of e-commerce lies in its convenience and low cost; it is implemented by the Internet. Enterprises use network resources to deliver goods and enterprise information to end customers, and to achieve cross-space services and differentiated services for customers. The modern e-commerce model covers the entire supply chain business flow, logistics, information flow, and capital flow, and is an extension of traditional retail and business service functions. Ecommerce has transformed the organization's organizational development from the original refined direction to a more advanced informationization direction. Logistics distribution under the e-commerce model refers to the logistics enterprises relying on the modern information network to support the strict, timed, fixed-point and trustworthy door-to-door logistics distribution for consumers of different regions and different requirements with advanced management methods. This includes the necessary packaging, sorting, sorting, distribution, delivery, etc. that occur during the logistics process to meet the needs of customers [1]. Logistics distribution is the basis and support for the realization of e-commerce, and it is the link between virtual service and entity business flow. The level of logistics directly determines the level of e-commerce. Without advanced logistics assistance, there is no broader development of e-commerce and customer recognition [2]. The relationship between e-commerce and logistics distribution can be further analyzed from the following two aspects. First, logistics and distribution have become an essential part of e-commerce. The realization of e-commerce functions requires that it must include logistics distribution. Second, logistics and distribution are the guarantee of e-commerce. Strengthening the logistics distribution capacity is an inevitable requirement for the development of e-commerce and the enhancement of core competitiveness. At present, the e-commerce end express co-delivery method faces problems of low distribution efficiency and low customer satisfaction. It is necessary to study the co-delivery collaborative delivery method of ecommerce terminal [3].

* Corresponding author.

E-mail address: 25280480@qq.com 
Kang et al. proposed a method for optimizing the delivery route of e-commerce terminals. The method considered the delivery time, service satisfaction and distribution cost, and constructed a joint distribution path optimization model with a time window. They proposed an adaptive improved genetic algorithm, which uses PFIH algorithm to construct the initial solution, novel mutation operator and adaptive cross mutation probability, and the RCA algorithm to quantify multi-target weights. The optimal path of distribution is obtained. The method takes a long time to distribute, and there is the problem of low distribution efficiency. Sun et al. proposed a logistics distribution method based on a hybrid genetic algorithm. This method constructed a mathematical model of logistics distribution path optimization and improved global convergence performance by selecting niche technology based on local competition mechanism and adaptively adjusting cross-variation parameters. The simulated annealing algorithm with memory function was combined with the genetic algorithm to complete the coordinated delivery of the e-commerce end express. This method cannot meet the customer's multiple distribution requirements, and there is a problem of low customer satisfaction [4]. Li et al. proposed an e-commerce-based co-delivery collaborative delivery method based on the Internet of Things. According to the advantages of the Internet of Things technology and the characteristics of express delivery, this method introduced main constraints such as fuzzy time window function, customer satisfaction function and express loss function. The multi-objective path optimization model in the Internet of Things environment with the goal of maximum customer satisfaction and minimum distribution cost was constructed. The final path of the method was that the probability of the optimal path was low, the delivery time was long, and there is a problem of low distribution efficiency [5]. Qian et al. proposed a hybrid delivery simulation method based on e-commerce terminal express delivery. The method considered the fixed cost, variable distribution cost and delivery mileage of each vehicle, and established a multi-vehicle vehicle path optimization model with a fixed number of vehicles with the goal of the minimum total cost. The multi-operator neighborhood search based on probability was introduced. The simulated annealing algorithm was improved by the taboo table in tabu search, and the coordinated delivery of e-commerce end express was completed. This method faces the problem of low customer satisfaction [6].

In order to solve the problems in the above methods, a method based on big data platform for e-commerce end express co-delivery is proposed. Specific steps are as follows.

(1) Select the distribution center of the e-commerce end express by using the location selection method based on the component analysis.

(2) Establish a courier collaborative delivery path optimization model based on customer satisfaction.

(3) Experimental results and analysis. The overall effectiveness of the E-commerce end-courier collaborative delivery method based on big data platform is verified in terms of distribution efficiency and customer satisfaction.

(4) Conclusion.

\section{Characteristics of E-Commerce End Express Co-Delivery}

The e-commerce model has its own characteristics; the e-commerce end express co-delivery flow chart is shown in Figure 1.

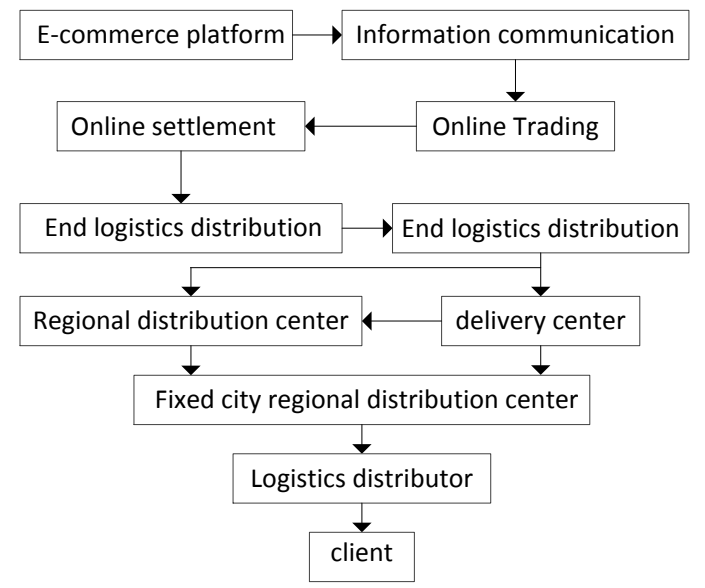

Figure 1. Schematic diagram of courier delivery at the end of e-commerce

(1) Characteristics of e-commerce terminal logistics. First: small batch size and small packaging. Second, customer 
demand is random. Third, timing is fixed, and delivery timeliness and integrity requirements are high. Fourth, the demand for return logistics is high. Fifth, the ability to track the goods in transit is high, and the customer can be inquired at any time. Sixth, the logistics capacity to meet the above customer needs and logistics distribution requirements is high.

(2) The actual demand for co-delivery delivery at the end of e-commerce. Based on the above analysis, the demand for end-to-cloud co-delivery is focused on the two themes of "customer" and "enterprise". The analysis is as follows.

The customer's demand for express delivery at the end of e-commerce includes two aspects. On the one hand, demand needs to reflect the cost advantage. Customers always want to buy more affordable products that are "cheap", which means low logistics and low cost while maximizing cost advantage. On the other hand, demand needs to reflect convenience. The customer requires logistics and distribution to continue and fluently as e-commerce and achieve door-to-door delivery services according to the time and place required by customers.

The demand for express delivery of e-commerce is at the end of the enterprise. We must continue the corporate brand and image. That is to say, enterprises require logistics distribution, even for third-party logistics and distribution, to represent the image and brand of the company to a certain extent, reflecting the corporate service concept and performance represented by the brand image. On the other hand, it meets the needs of the company. When enterprises adopt marketing methods such as a promotion for their own interests, especially when customers increase greatly, they need logistics capabilities and levels to meet the needs of enterprises and cooperate with corporate marketing strategies to achieve the desired results. In addition, it should also meet the customer's diverse receiving needs. Convenience is one of the core competitiveness elements of e-commerce companies. Enterprises must require logistics to continue their service concept at the end of the distribution process and meet the needs of customer convenience with a variety of flexible services [7].

\section{E-Commerce End Express Co-Delivery Distribution Method based on the Big Data Platform}

\subsection{Site Selection based on Component Analysis}

Based on the big data platform, the e-commerce end express co-delivery method uses the component selection method based on component analysis to select the distribution center. The distribution cost model is obtained through inventory cost, fixed investment cost, storage cost, and operation cost. The total cost corresponding to all candidate solutions is compared based on the total cost corresponding to all the scenarios. The candidate scheme corresponding to the minimum total cost is selected as the final multi-distribution center location scheme under e-commerce.

Transportation costs include costs from the point of supply to the distribution center, and distribution costs from the distribution center to the demand point [8]. The candidate program is brought into the cost model of the distribution center location factor under the e-commerce. Inventory, warehousing, fixed investment, and operation cost corresponding to the candidate solution are obtained, and the total cost corresponding to the candidate solution is calculated. Let $T C$ represent the cost model, and the expression of $T C$ is as follows.

$$
T C=C I+C S+C F+C O
$$

Where, $T C$ is the total cost $T C$; $C I$ is the inventory cost; $C F$ is the fixed investment cost, $C S$ is the storage cost, and $C O$ is the operating cost.

For the inventory cost of the candidate solution, it is necessary to correspond to the logistics mode under e-commerce. Goods in the third-party logistics model are not owned by the distribution center. Therefore, there is no inventory cost, and only the inventory cost exists in the e-commerce self-built logistics mode. Combining the demand of the demand point, the inventory quantity of the distribution center, and the supply quantity and the supply time of the supply point, the average inventory of the distribution center is calculated. The calculation formula of the inventory cost $C I$ is as follows.

$$
C I=\sum_{p=1}^{P} \sum_{h=1}^{H} I_{h} E_{h}\left(\left(T \sum_{n=1}^{N} Y_{p n} u_{h n}+k_{h p} \sqrt{L_{h p}}\left(\sqrt{\sum_{n=1}^{N} Y_{p n} \sigma_{n h}^{2}}\right)\right) / 2\right)
$$

In the formula, $p$ is the distribution center parameter, the distribution center $p$ obeys $N\left(\mu_{h p}, \sigma_{h p}^{2}\right) ; h$ is the product parameter; $I_{h}$ is inventory cost factor; $E_{h}$ is cost-based price parameter; $T$ is maximum inventory; $u_{h n}$ is customer demand 
parameter; $k_{h p}$ is distribution center safety stock indicator; $L_{h p}$ the distribution center that replenishment lead time parameters for delivery of goods; $n$ is customer parameter; $Y_{p n}$ is integer variable with a range of $0-1$; the parameters satisfy the conditions $\sigma_{n h}^{2}=\sigma_{h p}^{2} / Y_{p n}$.

The storage cost corresponding to the candidate scheme also needs to consider the logistics mode under e-commerce; only the third-party logistics model has storage cost [9]. Calculate the storage cost, mainly considering the demand of the demand point, to obtain the average storage of the distribution center. Combined with the storage rate, the storage cost of the distribution center $C S$ in the candidate solution can be obtained. $C S$ can be calculated by Equation (3).

$$
C S=\sum_{p=1}^{P} \sum_{h=1}^{H} V_{p h} E_{h}\left(\left(\sum_{n=1}^{N} Y_{p n} u_{h n}+k_{p h} \sqrt{L_{h p}}\left(\sqrt{\sum_{n=1}^{N} Y_{p n} \sigma_{n h}^{2}}\right)\right) / 2\right)
$$

where $V_{p h}$ is storing rates for products stored $h$ in distribution centers $p$.

By analyzing the influencing factors of the macro environment on the location of the distribution center, the calculation formula of the fixed investment cost $C F$, corresponding to the candidate scheme, is as follows.

$$
C F=\sum_{P=1}^{P} f_{p} Z_{p}+\sum_{P=1}^{P} I N T_{P} Z_{p}
$$

In the formula, $f_{p}$ is the fixed investment cost rate when the distribution center $p$ is delivered; $Z_{p}$ is the integer variable of $0-1 ; I N T_{P}$ is the fixed investment cost rate coefficient of the distribution center network under e-commerce.

Through government policy, human factors, customer distribution, supplier distribution, human resource cost and other factors, the calculation formula of the operating cost corresponding to the candidate scheme $C O$. $C O$ is as follows.

$$
C O=\sum_{P=1}^{P} Q_{P} M_{P}+\sum_{P=1}^{P} R_{p m} N N_{p m}+\sum_{P=1}^{P} R_{p o} N N_{p o}+\sum_{P=1}^{P} U_{p}+\sum_{P=1}^{P} D_{p}
$$

In the formula, $Q_{P}$ is the distribution center management cost rate; $M_{P}$ is the distribution center construction scale index; $R_{p m}$ is the salary rate of the management staff of the distribution center; $N N_{p m}$ is the total number of management personnel of the distribution center; $R_{p o}$ is the salary rate of the operator; $N N_{p o}$ is the total number of operators; $U_{p}$ is the water, electricity and energy costs near the distribution center; $D_{p}$ is the tax payment for the distribution center.

The inventory cost, the storage cost, the fixed investment cost, and the operation cost of the distribution center corresponding to the candidate solution obtained by the above calculation are added and calculated. The total cost of the distribution center under the e-commerce of each candidate solution is obtained. Choose the distribution center with the lowest total cost as e-commerce.

\subsection{E-Commerce End Express Co-Delivery}

\subsubsection{Description of the Problem}

The problem of distribution path optimization based on customer satisfaction can be described as follows. There is a distribution center with several transportation vehicles. These vehicles start from the distribution center and serve customers who are bound by different geographical locations, the demand for goods and the allowed service time. Each customer can only serve one car, but one car can serve multiple customers. Find the driving route of the vehicle that meets the customer's distribution needs and has the highest customer satisfaction under certain cost conditions. Assume that the speed of the vehicle is fixed and the service time of each customer is fixed. The service time period that each customer can accept and the fully satisfactory service time period is set. The shortest distance between the customer and the customer, and between the customer and the distribution center is established [10]. 
Traditional path optimization problems are mostly aimed at minimizing transportation costs or minimizing transportation time. With regards to the idea of customer satisfaction based on the big data platform, the e-commerce end express co-delivery distribution method combines the traditional goal. The goal of the e-commerce end express co-delivery problem under the large number platform is reduced to three points. They are maximizing customer satisfaction while minimizing vehicle waiting time and vehicle transportation costs [11].

In order to accurately reflect the customer satisfaction status of the distribution center and master the dynamic changes in customer needs, it is necessary to evaluate and optimize customer satisfaction [12]. From the perspective of customer satisfaction, customers may prefer to start delivering services at a specific point in time or time period. Services that start at other time points or time periods will cause inconvenience to customers and reduce customer satisfaction. The most important factor affecting customer satisfaction in the co-delivery of e-commerce at the end of the e-commerce is the punctuality of delivery, and customer satisfaction is described by the punctuality of delivery. According to the above analysis, it is necessary to define the scope of the delivery time of the customer's demand when selecting the delivery route, so that the satisfaction of the delivery within the time range is the highest in the distribution process [13]. Based on the big data platform, the e-commerce end express co-delivery method introduces the concept of time window to solve the problem of delivery time range.

The time window is a time period $[S t, E t]$, which is a service time interval determined by the earliest service time $S t$ and the latest service time $E t$ requested by the customer. At present, the research on time windows can be generally divided into three categories: hard time windows, soft time windows and hybrid time windows [14]. The three are described as follows.

(1) Hard time window: Each product delivery task must be completed within the required time range, as shown in Figure 2.

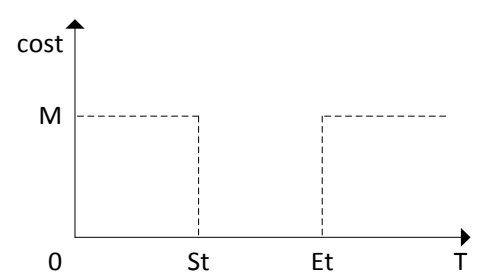

Figure 2. Schematic diagram of hard time window

The customer will not receive the service in advance or postponed, whether it is early or late. The delivery vehicle arrives in advance and must wait until the earliest start of service time in order to provide services to the customer. The delivery vehicle arrives late and the customer refuses to accept the delivery service.

(2) Soft time window: Each task cannot be completed within the required time range, as shown in Figure 3.

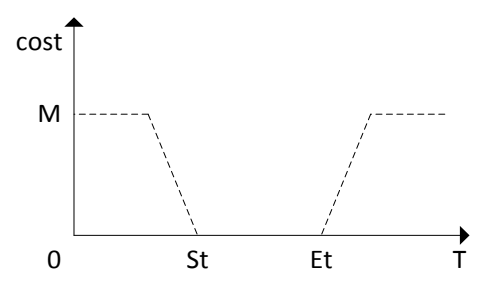

Figure 3. Soft time window schematic

Analysis of Figure 3 shows that the customer accepts the delivery service outside of the time window. The delivery vehicle arrives within the delivery time requested by the customer. Distribution is the most punctual and the service level is optimal. When the time window of the customer's request is violated, the customer accepts the service, but the delivery service level decreases and the customer satisfaction decrease.

(3) Hybrid time window: Some customers in the system belong to hard time windows, while others belong to soft time windows. The same customer often mixes both soft and hard time windows, as shown in Figure 4. 


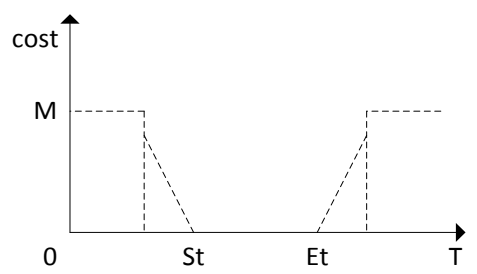

Figure 4. Schematic diagram of the mixed time window

In the actual logistics distribution, if the delivery vehicle can implement the delivery service at the delivery time requested by the customer, the delivery service level is optimal, and the customer satisfaction is the best. If the delivery vehicle implements the delivery service within a period of time beyond the service time, the customer's satisfaction is reduced, and the customer does not accept the time delivery service beyond the above time periods.

\subsubsection{Description of the Problem}

In an increasingly competitive environment, in order to better maintain the cooperation between the distribution center and the customers, we can deliver and service the customers within the specified time, improve competitiveness and achieve maximum customer satisfaction [15]. This is especially important in today's society. Considering that distribution centers need to use limited resources to serve large-scale customers, it is difficult to make all customers fully satisfied. In the process of delivery, customer's specified location in advance will need to wait until the earliest start of service so that the customer will be satisfied. The corresponding cost will be waiting [16]. When the delivery vehicle arrives at the customer's specified location, the customer satisfaction will be reduced. The time window is shown in Figure 5.

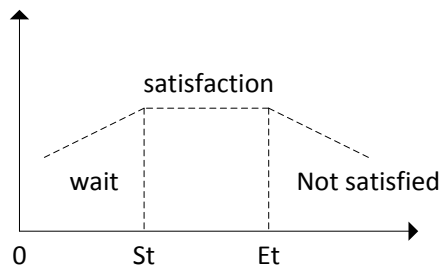

Figure 5. Schematic diagram based on customer satisfaction time window

It can be seen that both the customer satisfaction function and the vehicle waiting cost function are related to the arrival time of the delivery. The costs caused by waiting for the opportunity cost and the customer's dissatisfaction are respectively on both sides of the delivery specified time. Combined with the actual situation analysis, the multi-objective model is processed by penalizing the violation of the time window, making its solvability easier to achieve. In general, the penalty amount of the distribution center is set according to the contract signed between the distribution center and the customer, and the penalty cost is proportional to the difference between the delivery vehicle and the specified delivery time. That is, the greater the offset between the delivery vehicle and the fixed time, the higher the penalty cost. Establish a penalty function

$$
P(k, n)=\left\{\begin{array}{l}
\left.c_{1}(E t(n)-T(k, n)), T(k, n)\right)<E t(n) \\
0, E t(n)<T(k, n)<L t(n) \\
c_{2}(T(k, n)-L t(n)), L t(n)<T(k, n)
\end{array}\right.
$$

where $P(k, n)$ is the penalty function value delivered by the $k^{\text {th }}$ vehicle to the $n^{\text {th }}$ customer; $\operatorname{Et}(n)$ is the earliest time allowed for the vehicle to enter the station; $T(k, n)$ is the time when the $k^{\text {th }}$ vehicle arrives at the $n^{\text {th }}$ customer; $L t(n)$ is the latest time to allow the vehicle to enter the station; $c_{1}$ is the opportunity cost of waiting for the unit time at the mission point; $c_{2}$ is the penalty value at which the vehicle arrives at the unit time after the required time.

Among them, the size of $c_{1}$ and $c_{2}$ in the penalty function can be determined according to the actual operation of the distribution industry. If the vehicle arrives at customer $n$ before $E t(n)$, a waiting cost $c_{1}(E t(n)-T(k, n))$ is generated. If the delivery vehicle arrives at customer $n$ after $\operatorname{Lt}(n)$ o'clock, the customer is not satisfied with the delivery service level, 
and the penalty cost is $c_{2}(T(k, n)-L t(n))$. If the delivery vehicle arrives between the delivery service time window $[\operatorname{Et}(n), \operatorname{Lt}(n)]$ requested by the customer, the customer satisfaction is the highest, the waiting cost is the smallest, and the penalty cost is zero. A schematic diagram of the penalty function can be obtained as shown in Figure 6.

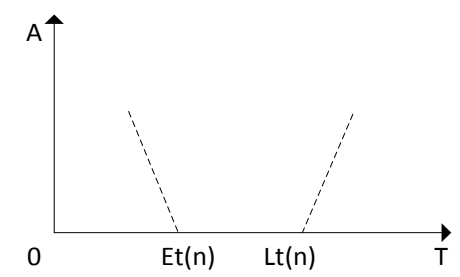

Figure 6. Schematic diagram of the penalty function

Among them, $A$ is the penalty cost of the distribution process. The penalty cost $A$ for all vehicles to deliver to customers can be expressed as Equation (7).

$$
A=c_{1} \sum_{n=1}^{N} \max [\operatorname{Et}(n)-T(n), 0]+c_{2} \sum_{n=1}^{n} \max [T(n)-L t(n), 0]
$$

\subsubsection{Description of the Problem}

From the above analysis, it can be clearly concluded that the path optimization model based on the big data platform-based e-commerce end express co-delivery method can be composed of two parts: the penalty cost of collaborative distribution cost and customer satisfaction; the total cost is the sum of these two parts. The expression of path optimization model $C$ is as follows.

$$
C=Z+A
$$

In the formula, $Z$ is the cost of co-delivery delivery at the end of the e-commerce. Through the path optimization model, the optimal route of the e-commerce end express co-delivery is obtained, and the coordinated delivery of the ecommerce end express is completed.

\section{Experimental Results and Discussion}

In order to verify the overall effectiveness of the e-commerce terminal express delivery collaborative distribution method based on big data platform, it is necessary to test the e-commerce end express co-delivery distribution method based on big data platform. The experimental platform for this test was Mulan and the operating system were Windows 7.0. The ecommerce end-coupon co-delivery distribution method based on the big data platform (method 1), the hybrid genetic algorithm-based logistics distribution method (method 2), and the internet of things-based e-commerce end express codelivery method (method 3). These three methods cooperatively distribute the express delivery of five customers. The distribution route of three different methods is shown in Figure 7.

Figure 7(a) is a distribution path of the e-commerce end express co-delivery method based on the big data platform. Figure 7(b) shows the distribution path of the logistics distribution method based on the hybrid genetic algorithm. Figure 7(c) is a distribution path of an e-commerce-based express delivery cooperative distribution method based on the Internet of Things. Analysis of the three figures shows that there is no duplicate link in the delivery path of the e-commerce end express co-delivery method based on the big data platform. Figure 8 shows the time taken for the collaborative delivery of five customers' couriers by three different methods.

Figure 8(a) shows the time taken to deliver five customers' express delivery based on the big data platform's ecommerce end express co-delivery method. Analysis of Figure 8(a) shows that the delivery time used in this method is within one hour. Figure 8(b) shows the time taken to deliver the five customers' express delivery based on the hybrid genetic algorithm. Figure 8(c) shows the time taken to deliver the five customers' express delivery based on the Internet of Thingsbased e-commerce end express co-delivery method. Analysis of Figure 8(b) and Figure 8(c) shows that the delivery times used in the above two methods are as high as $1.3 \mathrm{~h}$ and $1.7 \mathrm{~h}$, respectively. Comparing the test results of three different methods, it can be known that the e-commerce end-coupon collaborative distribution method based on the big data platform 
uses a short delivery time and high distribution efficiency.

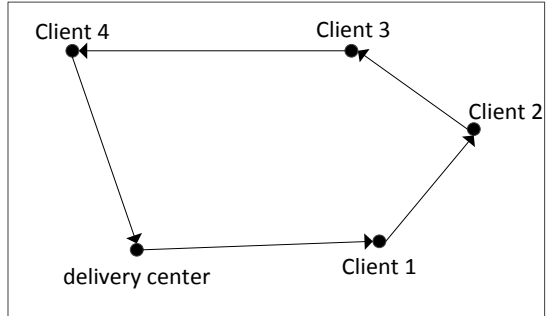

(a) Method 1 of the delivery path

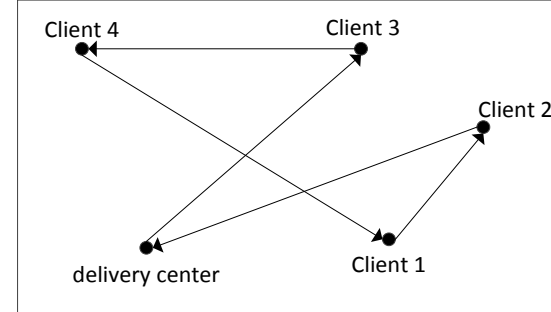

(b) Delivery path of method 2

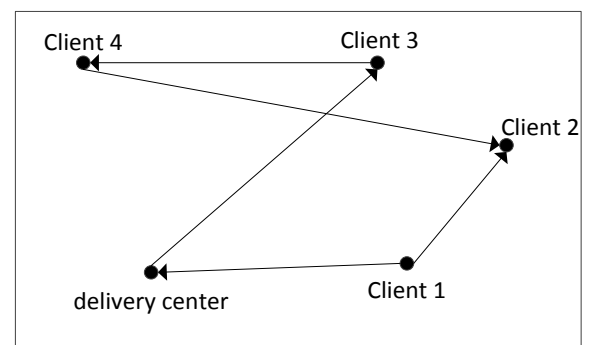

(c) Delivery path of method 3

Figure 7. Distribution path of three different methods

In order to further verify the overall effectiveness of the E-commerce end-coupon collaborative delivery method based on the big data platform, five customers were invited to score the three methods. The three methods are as follows: ecommerce end-coupon co-delivery method based on big data platform (method 1), hybrid genetic algorithm-based logistics distribution method (method 2), and e-commerce-based e-commerce end express co-delivery method (method 3). Compare the customer satisfaction of the three different methods; the test results are shown in Figure 9.

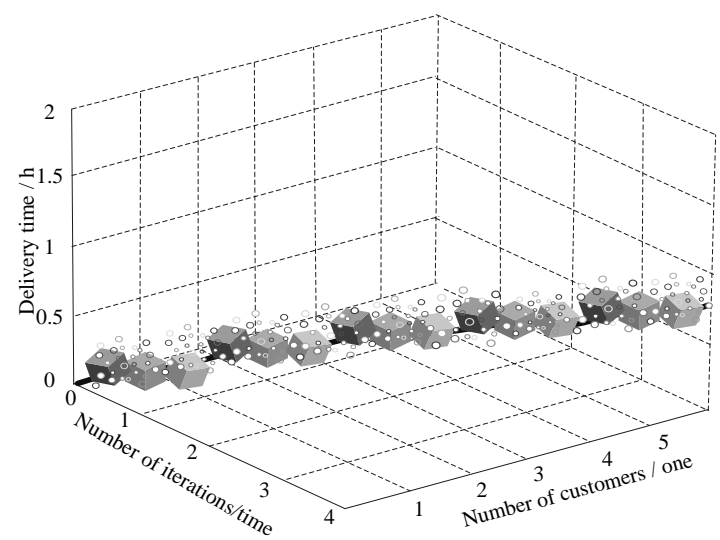

(a) Delivery time of method 1

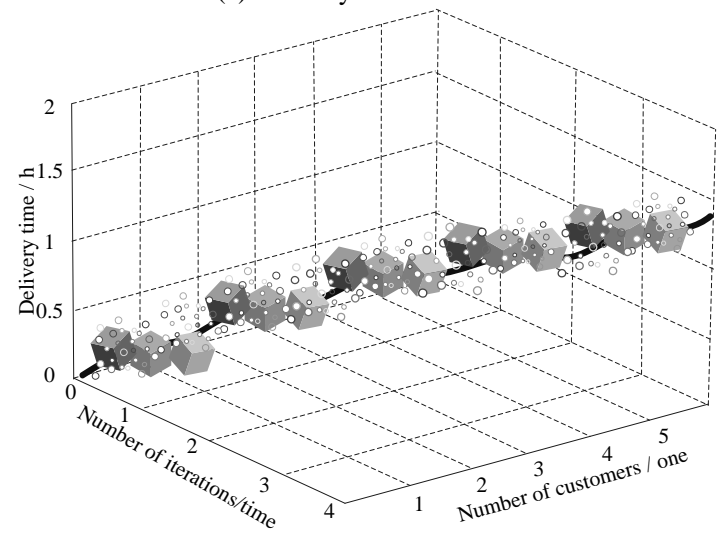

(b) Delivery time of method 2 


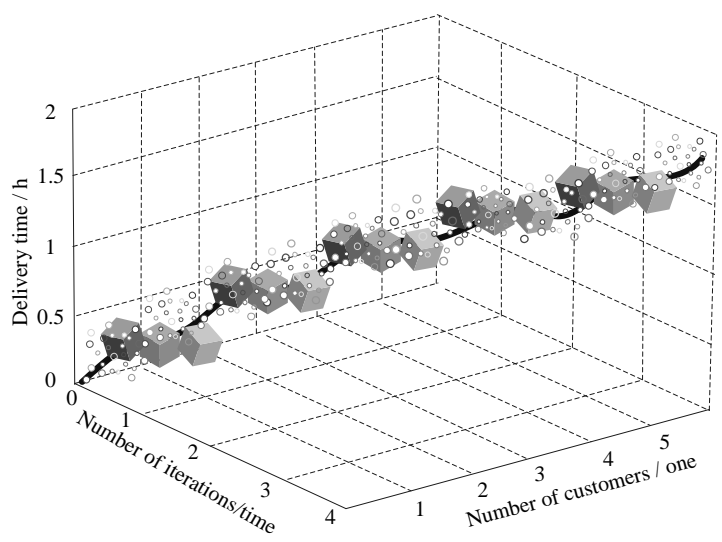

(c) Delivery time of method 3

Figure 8. Delivery time for three different methods

Figure 9(a) shows the customer satisfaction of the E-commerce end-coupon collaborative delivery method based on the big data platform. Analysis of Figure 9(a) shows that the satisfaction of the five customers is above 80\%. Figure 9(b) shows the customer satisfaction of the logistics distribution method based on a hybrid genetic algorithm. Analysis of Figure 9(b) shows that the highest satisfaction achieved by this method is $60 \%$. Figure 9 (c) is the customer satisfaction of the ecommerce-based e-commerce end express co-delivery method. Analysis of Figure 9(c) shows that the highest satisfaction rate obtained by this method is $50 \%$. By comparing the test results of three different methods, customer satisfaction of the ecommerce end express co-delivery method based on big data platform is higher.

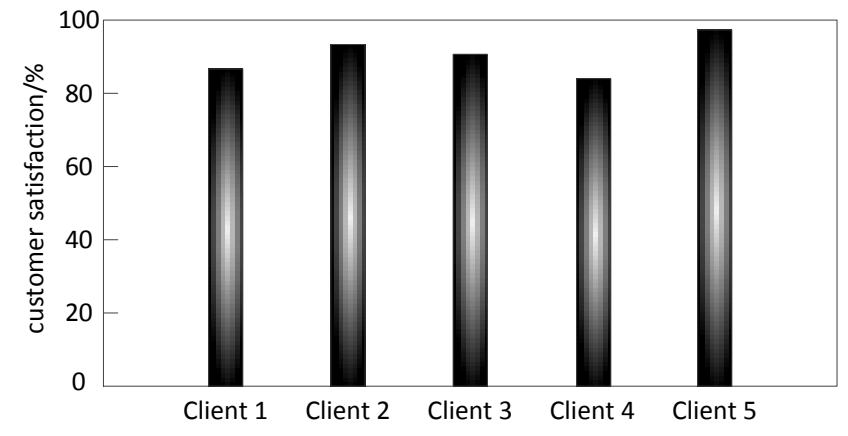

(a) Customer satisfaction in Method 1

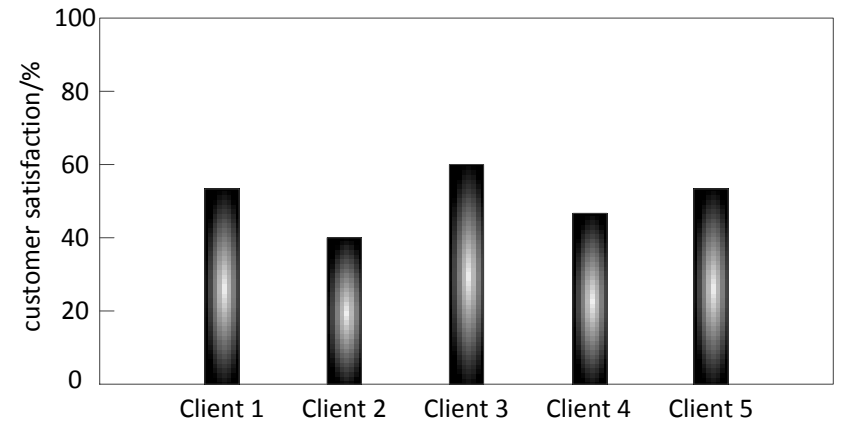

(b) Customer satisfaction in Method 2

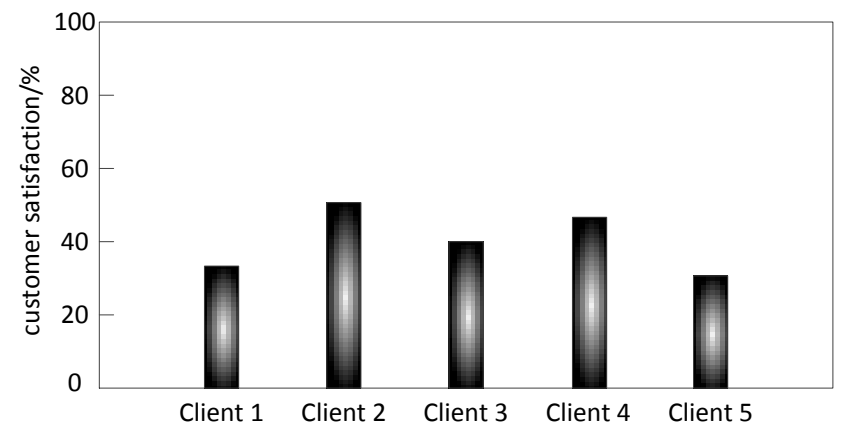

(c) Customer satisfaction in Method 3

Figure 9. Customer satisfaction of three different methods

\section{Conclusion}

Today's logistics has developed to a relatively high level, but distribution centers often have problems of high cost and serious waste of resources when distributing. Some distribution channels even have more isolated distribution customers, which limits the development of logistics today. Reduction of distribution costs, resource utilization and distribution efficiency are urgent problems to be solved. At present, the e-commerce end express co-delivery method faces problems of low distribution efficiency and low customer satisfaction and proposes a co-delivery collaborative delivery method based on big data platform. This solves the problems in the current method and promotes the development of e-commerce. 


\section{References}

1. L. Xie, W. Zhou, and Y. Li, "Application of Improved Recommendation System based on Spark Platform in Big Data Analysis," Cybernetics and Information Technologies, Vol. 16, pp. 245-255, 2016

2. A. G. Roesener and J. W. Barnes, "An Advanced Tabu Search Approach to the Dynamic Airlift Loading Problem," Logistics Research, Vol. 9, pp. 12, 2016

3. F. Zhu and S. V. Ukkusuri, "A Linear Programming Formulation for Autonomous Intersection Control Within a Dynamic Traffic Assignment and Connected Vehicle Environment," Transportation Research Part C: Emerging Technologies, Vol. 55, pp. 363-378, 2015

4. M. Sun and D. Pang, "Vehicle Routing Optimisation Algorithm for Agricultural Products Logistics Distribution," International Journal of Applied Decision Sciences, Vol. 10, pp. 327-334, 2017

5. C. B. Li, E. J. Wang, and J. B. Yuan, "Research on the Optimization of Logistics Distribution Route of Fresh Agricultural Produce under Internet of Things," Commercial Research, Vol. 4, pp. 001, 2017

6. S. Liu, W. Fu, W. Zhao, J. Zhou, and Q. Li, "A Novel Fusion Method by Static and Moving Facial Capture," Mathematical Problems in Engineering, Vol. 2013, pp. 503924, 2013

7. S. Jeong, O. Simeone, and J. Kang, "Mobile Edge Computing via a UAV-Mounted Cloudlet: Optimization of Bit Allocation and Path Planning," IEEE Transactions on Vehicular Technology, Vol. 67, pp. 2049-2063, 2018

8. P. Caputa, N. Kundu, M. Miyaji, T. Takayanagi, and K. Watanabe, "Anti-De Sitter Space from Optimization of Path Integrals in Conformal Field Theories," Physical Review Letters, Vol. 119, pp. 071602, 2017

9. O. B. Haddad, P. S. Ashofteh, and M. A. Marino, "Levee Layouts and Design Optimization in Protection of Flood Areas," Journal of Irrigation and Drainage Engineering, Vol. 141, pp. 04015004, 2015

10. C. K. Chau, K. Elbassioni, and C. M. Tseng, "Drive Mode Optimization and Path Planning for Plug-in Hybrid Electric Vehicles," IEEE Transactions on Intelligent Transportation Systems, Vol. 18, pp. 3421-3432, 2017

11. G. Yang and S. Liu, "Distributed Cooperative Algorithm for k-M Set with Negative Integer k by Fractal Symmetrical Property," International Journal of Distributed Sensor Networks, Vol. 10, pp. 398583, 2014

12. J. Berger, N. Lo, and M. Barkaoui, "Static Target Search Path Planning Optimization with Heterogeneous Agents," Annals of Operations Research, Vol. 244, pp. 295-312, 2016

13. V. Kungurtsev and J. Jaschke, "A Predictor-Corrector Path-Following Algorithm for Dual-Degenerate Parametric Optimization Problems," SIAM Journal on Optimization, Vol. 27, pp. 538-564, 2017

14. E. Tzoreff and A. J. Weiss, "Single Sensor Path Design for Best Emitter Localization via Convex Optimization," IEEE Transactions on Wireless Communications, Vol. 16, pp. 939-951, 2017

15. Z. Pan, S. Liu, and W. Fu, "A Review of Visual Moving Target Tracking," Multimedia Tools \& Applications, Vol. 76, pp. 16989-17018, 2017

16. X. He, Y. Zhou, and Z. Chen, "An Evolution Path based Reproduction Operator for Many-Objective Optimization," IEEE Transactions on Evolutionary Computation, Vol. 23, No. 1, pp. 29-43, 2017 